\title{
Energy content of stools in normal healthy controls and patients with cystic fibrosis
}

\author{
J L Murphy, S A Wootton, S A Bond, A A Jackson
}

\begin{abstract}
Stool energy losses and the sources of energy within the stool were determined in $\mathbf{2 0}$ healthy controls and 20 patients with cystic fibrosis while on their habitual pancreatic enzyme replacement treatment. Stool energy losses were equivalent to $3.5 \%$ of gross energy intake in healthy children (range $\mathbf{1 \cdot 3 - 5 \cdot 8 \%}$ ). Despite a comparable gross energy intake, stool energy losses were three times greater in patients with cystic fibrosis than controls averaging $10.6 \%$ of gross energy intake (range 4.9-19.7\%). Stool lipid could account for only $29 \%$ and $41 \%$ of the energy within the stool in controls and patients with cystic fibrosis respectively and was poorly related to stool energy. Approximately $30 \%$ of the energy within the stool could be attributable to colonic bacteria in both the healthy children and patients with cystic fibrosis. These results suggest that stool energy losses in healthy children are relatively modest but that even when patients with cystic fibrosis are symptomatically well controlled on pancreatic enzyme replacement, raised stool energy losses may continue to contribute towards an energy deficit sufficient to limit growth in cystic fibrosis. As the energy content per gram wet weight remains relatively constant $(8 \mathrm{~kJ} / \mathrm{g})$, stool energy losses may be estimated from simple measurements of stool wet weight.
\end{abstract}

Department of
Human Nutrition,
University of
Southampton,
Bassett Crescent East,
Southampton SO9 3TU
J L Murphy
S A Wootton
S A Bond
A A Jackson
Correspondence to:
Dr Wootton.

The amount of energy within the stool each day in healthy adults is generally believed to be equivalent to approximately $5 \%$ of the gross energy intake. ${ }^{1}$ As stool energy losses appear to be relatively small and the collection and analysis of faecal material is both time consuming and unpleasant, the potential contribution that stool energy losses may make in limiting the availability of energy from the diet is often overlooked. To our knowledge, there have been no studies that have been specifically conducted to determine the magnitude of stool energy losses in children aged between 5-16 years. Our understanding of both the magnitude and origins of energy within the stool in normal healthy children and diseased states in childhood is limited. Further studies are required to determine whether stool energy losses may limit the availability of energy from the diet and thereby restrict growth.

One disease where the maldigestion and malabsorption of lipid, protein, and carbohydrate may limit the availability of energy from the diet resulting in restricted growth is cystic fibrosis. ${ }^{2}$ The standard approach to the management of the gastrointestinal problems associated with cystic fibrosis has been exogenous pancreatic enzyme replacement treatment. In clinical practice, enzyme replacement is primarily directed towards the symptomatic correction of steatorrhoea, abdominal pain relief, and the reduction in stool frequency and stool mass passed each day. Of these, steatorrhoea would appear to be the most important clinical consideration and has frequently been used as an index of the extent of maldigestion. ${ }^{34}$ Using this empirical approach it is commonly assumed that when the gastrointestinal symptoms and steatorrhoea are improved, the amount of energy available from the diet would be normal. However, the extent to which stool energy losses are completely normalised in patients with cystic fibrosis on their habitual established enzyme replacement has not been determined.

Therefore the aim of this study was to determine the amount of energy within the stools each day in normal healthy children and in patients with cystic fibrosis who believed themselves to be comparatively asymptomatic on their habitual pancreatic enzyme replacement treatment.

\section{Patients and methods}

Twenty normal healthy children aged 6-11 years (mean $9 \cdot 4$ years) from a local school participated in the study. Twenty patients with cystic fibrosis aged 5-25 years (mean $11 \cdot 4$ years) from a cystic fibrosis clinic in the Wessex region were also studied on their normal established pancreatic enzyme replacement therapy. Subject details are shown in the table. The patients with cystic fibrosis were taking between 11-43 (mean 22) capsules of pancreatin (Creon, Duphar: 8000 BP units lipase/capsule) each day and had been taking the enzyme in the form of enteric coated microspheres for at least one year before the study. All of the patients self titrated their enzyme dosage against gastrointestinal symptoms including abdominal pain, diarrhoea, offensive bulky stools, and increased stool frequency. No attempt was made to alter or intervene with the management of the enzyme replacement therapy. Informed consent was obtained from all of the subjects and the study protocol was approved by the ethical committee of Southampton and South West Hampshire Health Authority.

Weighed food intake was recorded for a period of seven days in a standard manner as described by Marr $^{5}$ using digital electronic 
Details of the normal healthy controls and patients with cystic fibrosis including stool energy losses and gross energy intake

\begin{tabular}{|c|c|c|c|c|c|c|c|}
\hline $\begin{array}{l}\text { Subject } \\
\text { No }\end{array}$ & $\begin{array}{l}\text { Age } \\
\text { (years) }\end{array}$ & Sex & $\begin{array}{l}\text { Weight } \\
(\mathbf{k g})\end{array}$ & $\begin{array}{l}\text { Height } \\
(\mathrm{cm})\end{array}$ & $\begin{array}{l}\text { Gross energy } \\
\text { (kf/day) }\end{array}$ & $\begin{array}{l}\text { Stool energy } \\
\text { (kf/day) }\end{array}$ & $\begin{array}{l}\text { Pancreatic } \\
\text { enzyme } \\
\text { replacement } \\
\text { treatment } \\
\text { (capsules } \\
\text { /day) }\end{array}$ \\
\hline $\begin{array}{l}1 \\
2 \\
3 \\
4 \\
5 \\
6 \\
7 \\
8 \\
9 \\
10 \\
11 \\
12 \\
13 \\
14 \\
15 \\
16 \\
17 \\
18 \\
19 \\
20\end{array}$ & $\begin{array}{r}6 \cdot 4 \\
8 \cdot 7 \\
9 \cdot 9 \\
9 \cdot 9 \\
8 \cdot 1 \\
8 \cdot 1 \\
11 \cdot 0 \\
10 \cdot 2 \\
11 \cdot 1 \\
11 \cdot 1 \\
7 \cdot 5 \\
8 \cdot 4 \\
9 \cdot 7 \\
10 \cdot 4 \\
7 \cdot 6 \\
10 \cdot 7 \\
10 \cdot 3 \\
8 \cdot 3 \\
11 \cdot 0 \\
10 \cdot 1\end{array}$ & $\begin{array}{l}\mathbf{F} \\
\mathbf{F} \\
\mathbf{F} \\
\mathbf{F} \\
\mathbf{M} \\
\mathbf{F} \\
\mathbf{M} \\
\mathbf{F} \\
\mathbf{F} \\
\mathbf{M} \\
\mathbf{F} \\
\mathbf{M} \\
\mathbf{M} \\
\mathbf{M} \\
\mathbf{F} \\
\mathbf{M} \\
\mathbf{F} \\
\mathbf{M} \\
\mathbf{M}\end{array}$ & $\begin{array}{l}25 \cdot 4 \\
30 \cdot 9 \\
30 \cdot 3 \\
25 \cdot 3 \\
25 \cdot 5 \\
27 \cdot 6 \\
35 \cdot 0 \\
25 \cdot 0 \\
36 \cdot 4 \\
47 \cdot 3 \\
25 \cdot 4 \\
28 \cdot 6 \\
33 \cdot 5 \\
38 \cdot 1 \\
25 \cdot 8 \\
38 \cdot 4 \\
35 \cdot 1 \\
30 \cdot 9 \\
35 \cdot 4 \\
36 \cdot 3\end{array}$ & $\begin{array}{l}\text { trols } \\
124 \cdot 7 \\
134 \cdot 9 \\
144 \cdot 2 \\
139 \cdot 6 \\
129 \cdot 5 \\
128 \cdot 8 \\
138.9 \\
133.8 \\
142 \cdot 0 \\
146.4 \\
135 \cdot 7 \\
134 \cdot 1 \\
136 \cdot 4 \\
147 \cdot 7 \\
131 \cdot 5 \\
152 \cdot 6 \\
148 \cdot 8 \\
139 \cdot 5 \\
138.7 \\
145 \cdot 3\end{array}$ & $\begin{array}{r}8690 \\
8808 \\
7306 \\
7412 \\
8710 \\
8951 \\
8807 \\
10621 \\
9865 \\
11191 \\
9609 \\
9152 \\
10108 \\
9179 \\
7698 \\
10438 \\
10803 \\
9051 \\
10379 \\
12492\end{array}$ & $\begin{array}{l}191 \\
363 \\
355 \\
160 \\
169 \\
186 \\
268 \\
412 \\
271 \\
647 \\
446 \\
123 \\
242 \\
525 \\
294 \\
506 \\
301 \\
288 \\
458 \\
530\end{array}$ & $\begin{array}{l}= \\
= \\
= \\
= \\
= \\
= \\
= \\
= \\
= \\
= \\
=\end{array}$ \\
\hline Mean (SEM) & $9.4(0.3)$ & & $31.8(1 \cdot 3)$ & $138 \cdot 7(1 \cdot 6)$ & $9464(292)$ & 337 (33) & \\
\hline $\begin{array}{r}1 \\
2 \\
3 \\
4 \\
5 \\
6 \\
7 \\
8 \\
9 \\
10 \\
11 \\
12 \\
13 \\
14 \\
15 \\
16 \\
17 \\
18 \\
19 \\
20\end{array}$ & $\begin{array}{r}6.9 \\
13 \cdot 8 \\
7 \cdot 9 \\
8 \cdot 9 \\
17 \cdot 4 \\
10 \cdot 2 \\
22 \cdot 9 \\
14 \cdot 9 \\
9 \cdot 5 \\
9 \cdot 0 \\
18 \cdot 0 \\
5 \cdot 3 \\
8 \cdot 5 \\
5 \cdot 5 \\
5 \cdot 4 \\
5 \cdot 8 \\
8 \cdot 1 \\
6 \cdot 3 \\
17 \cdot 4 \\
25 \cdot 7\end{array}$ & $\begin{array}{l}\mathbf{F} \\
\mathbf{F} \\
\mathbf{F} \\
\mathbf{M} \\
\mathbf{F} \\
\mathbf{F} \\
\mathbf{M} \\
\mathbf{M} \\
\mathbf{M} \\
\mathbf{F} \\
\mathbf{F} \\
\mathbf{M} \\
\mathbf{F} \\
\mathbf{M} \\
\mathbf{F} \\
\mathbf{F} \\
\mathbf{F} \\
\mathbf{F} \\
\mathbf{M} \\
\mathbf{F}\end{array}$ & $\begin{array}{l}\text { Patients } \\
20 \cdot 4 \\
26 \cdot 5 \\
28 \cdot 1 \\
34 \cdot 2 \\
38 \cdot 9 \\
21 \cdot 8 \\
45 \cdot 3 \\
53 \cdot 2 \\
29 \cdot 5 \\
28 \cdot 0 \\
43 \cdot 3 \\
20 \cdot 0 \\
24 \cdot 8 \\
17 \cdot 5 \\
18 \cdot 3 \\
18 \cdot 0 \\
34 \cdot 0 \\
20 \cdot 0 \\
58 \cdot 9 \\
44 \cdot 0\end{array}$ & $\begin{array}{l}\text { cystic fibrosis } \\
118.6 \\
142.0 \\
128.9 \\
131.8 \\
154.0 \\
133.0 \\
159.9 \\
166.9 \\
132.6 \\
131.2 \\
155.0 \\
112.8 \\
133.2 \\
108.5 \\
113.4 \\
109.1 \\
135.0 \\
114.2 \\
175.1 \\
165.3\end{array}$ & $\begin{array}{r}10580 \\
10752 \\
10831 \\
9589 \\
5904 \\
5652 \\
9853 \\
14755 \\
8558 \\
8371 \\
5771 \\
5895 \\
13954 \\
6632 \\
11689 \\
7285 \\
11389 \\
7763 \\
15907 \\
17543\end{array}$ & $\begin{array}{r}2084 \\
1092 \\
1058 \\
850 \\
716 \\
383 \\
1020 \\
1504 \\
1224 \\
1414 \\
963 \\
493 \\
944 \\
767 \\
575 \\
539 \\
1152 \\
1134 \\
898 \\
1014\end{array}$ & $\begin{array}{r}30 \\
37 \\
20 \\
11 \\
23 \\
20 \\
12 \\
30 \\
9 \\
19 \\
15 \\
27 \\
14 \\
16 \\
43 \\
16 \\
24 \\
25 \\
15 \\
23\end{array}$ \\
\hline Mean (SEM) & $11 \cdot 4(1 \cdot 4)$ & & $31 \cdot 2(2 \cdot 8)$ & $136 \cdot 0(4 \cdot 6)$ & 9934 (787) & $991(87)$ & $22(1 \cdot 9)$ \\
\hline Significance & NS & & NS & NS & NS & $\mathrm{p}<0.0001$ & - \\
\hline
\end{tabular}

NS, not significant.

scales (Hanson). The families of the subjects were instructed in the use of the scales and the recording of food intake before commencing the study. They were closely monitored and given support throughout the study period. After completion of the record, the family was interviewed to ensure that there were no omissions or errors. The diaries were coded according to the McCance and Widdowson food tables ${ }^{6}$ with additional data from the supplements to the food tables ${ }^{789}$ and manufacturers' information. The weighed food intake was analysed using a computerised food composition database (MicroDiet, University of Salford). Gross energy intakes were estimated by multiplying the intakes of lipid $(39.3 \mathrm{~kJ} / \mathrm{g}$ ), protein (as nitrogen $\times 6.25 ; 23.6 \mathrm{~kJ} / \mathrm{g})$, carbohydrate $(17.4$ $\mathrm{kJ} / \mathrm{g}$ ) and dietary non-starch polysaccharide $(17 \cdot 4 \mathrm{~kJ} / \mathrm{g})$ by their respective heats of combustion values. ${ }^{10}$

All stools were collected for the final three days between carmine markers. The stools were collected into polyethylene bags and immediately frozen. All the stool collections were complete. All of the subjects were cooperative, willing, and capable of following the details of the procedure. At no time was difficulty encountered nor any need found for modification of the procedure to accommodate the subjects. The pooled three day stools were weighed and homogenised with distilled water. Aliquots were then freeze dried overnight (Genevac) to a constant weight.

The Gompertz and Sammons modification ${ }^{11}$ of the method of Van de Kamer et $a l^{12}$ was employed to measure the lipid content of the wet stool. Total nitrogen analysis was performed by an automated Kjeldahl technique (Tecator) on a sample of the wet stool. The energy content of the stool was measured by ballistic bomb calorimetry ${ }^{13}$ (Gallenkamp) on a sample of freeze dried stool. The bacterial content of the dried stool was determined by repeated fractional centrifugation using the method developed by Stephen and Cummings. ${ }^{14} \mathrm{~A}$ freeze dried stool sample was resuspended in formylsaline and $10 \%$ sodium lauryl sulphate. The amount of bacteria within the bacterial fraction was confirmed by phase contrast microscopy using a haemocytometer (Weber Scientific International) to enable microscopic counting. Of the four fractions obtained by this method, over $99 \%$ of the stool bacteria could be recovered within the bacterial fraction. 
A portion of the dried bacterial mass from each stool sample was pooled to provide a representative sample of faecal bacteria for both the healthy controls and patients with cystic fibrosis. The energy per gram of stool bacteria for each of the pooled samples was determined by bomb calorimetry in an attempt to estimate the proportion of stool energy that may be attributed to stool bacteria.

Values are presented as mean (SEM). Statistical comparisons between the patients with cystic fibrosis and control subjects were performed using the Student's $t$ test and differences between means were considered significant where $\mathrm{p}<0.05$. Associations between variables were tested by the Pearson product moment correlation coefficient (R).

\section{Results}

The gross energy intakes and stool energy losses are shown in the table for both healthy controls and patients with cystic fibrosis. There was no significant difference in gross energy intakes for both the healthy control subjects and the patients with cystic fibrosis $(9.46(0.29)$ compared with 9.93 (0.79) $\mathrm{MJ} /$ day; this was not significant). In healthy control children stool energy averaged $0.34(0.03) \mathrm{MJ} /$ day, $3.5 \%$ of the gross energy intake (range $1 \cdot 3-5 \cdot 8 \%$; fig 1 ). In contrast, the stool energy losses were three
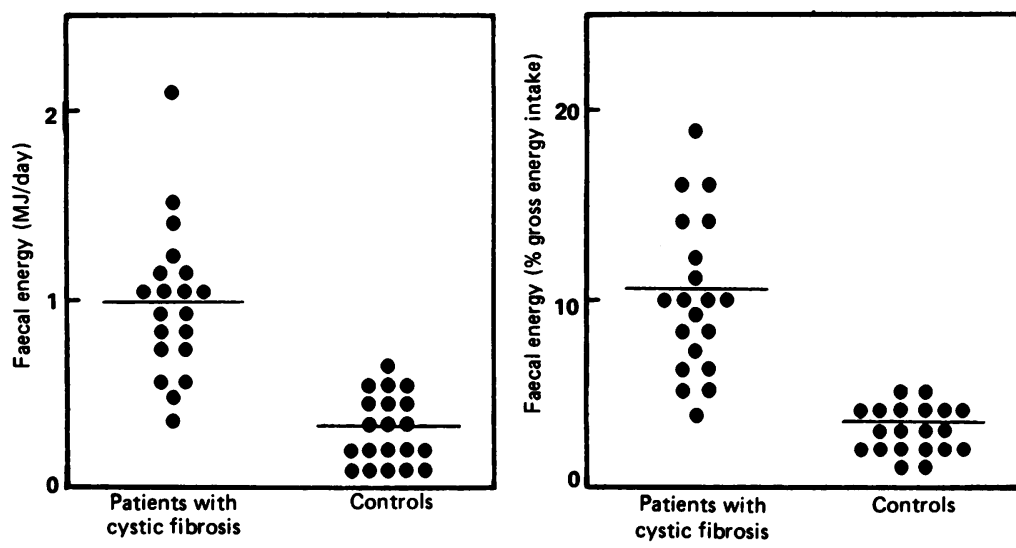

Figure 1 Daily stool energy losses expressed as MF/day and as a \% of the gross energy intake for each of the control subjects and cystic fibrosis patients. The bars represent the mean values. times greater in patients with cystic fibrosis than controls $(0.99(0.09) \mathrm{MJ} /$ day; $\mathrm{p}<0.0001)$, $10.6 \%$ of the gross energy intake (range 4.9 $19 \cdot 7 \% ; p<0.0001)$. In patients with cystic fibrosis, dry stool weight and wet stool weight were approximately twice as great as the controls (37.9 (3.6) compared with $16.8(1.8) \mathrm{g}$; $\mathrm{p}<0.0001$ and $120.8(9.8)$ compared with 69.3 $(8 \cdot 2) \mathrm{g} ; \mathrm{p}<0.001)$.

The amount of lipid within the stool of healthy control children averaged $2 \cdot 2(0 \cdot 2) \mathrm{g} /$ day, $2.9 \%$ of the lipid intake (range $1 \cdot 3-4 \cdot 4 \%$; fig 2 ). In patients with cystic fibrosis stool lipids were four times greater than in the controls $(9 \cdot 9(1 \cdot 2)$ g/day; $\mathrm{p}<0.0001$ ), equivalent to $14.6 \%$ of the lipids intake (range 2.8-37.9\%; $p<0.0001$ ). Nitrogen in the stool of healthy control children averaged $1 \cdot 1(0 \cdot 1) \mathrm{g} /$ day, $10.8 \%$ of nitrogen intake (range $4 \cdot 9-19 \cdot 1 \%$; fig 2 ). In patients with cystic fibrosis stool nitrogen was twice as great as the controls $(2.5(0.5)$ g/day; $\mathrm{p}<0.01)$, equivalent to $22 \cdot 5 \%$ of nitrogen intake (range 4.0-59.9\%; $\mathrm{p}<0.01)$.

There were weak associations between stool lipid and stool energy with only $26 \%$ and $19 \%$ of the variance in stool energy attributable to differences in stool lipid for both the control subjects and patients with cystic fibrosis respectively ( $R=0.51$ and $R=0.43$; fig 3 ). Stool lipid accounted for significantly more of the stool energy in the patients with cystic fibrosis $(41 \%)$ than healthy controls $(29 \% ; \mathrm{p}<0.05)$.

While stool lipid was a poor predictor of stool energy, a closer association could be demonstrated between stool energy and either the wet weight or the dry weight of the stool in both the control subjects $(R=0.77, R=0.98$ respectively; fig 4 and 5) and patients with cystic fibrosis $(R=$ $0.86, \quad R=0.98$ respectively). Approximately $59 \%$ and $74 \%$ of the variance in stool energy could be attributed to differences in wet stool weight in the control and cystic fibrosis patients respectively. For both groups, $96 \%$ of the variance in stool energy could be attributed to differences in dry stool weight.

The regression lines describing the association between the energy content and the dry weight of stool were similar for the control subjects and the patients with cystic fibrosis. For

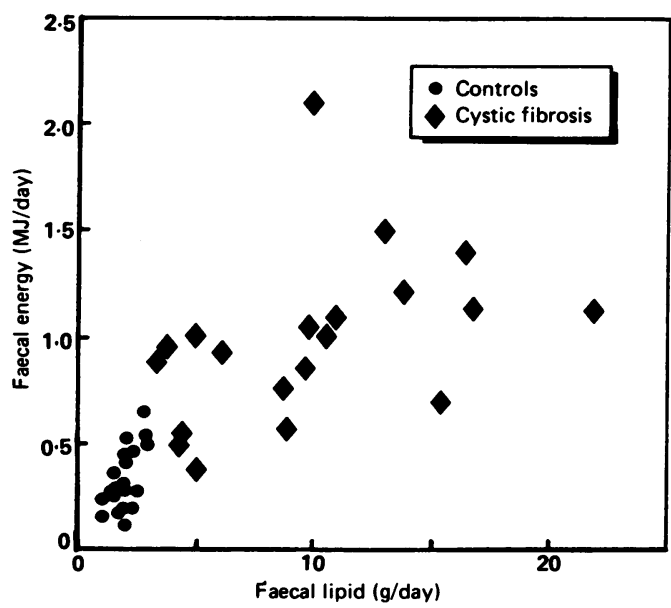

Figure 3 Stool energy versus stool lipid losses for the control subjects and cystic fibrosis patients.
Figure 2 Daily stool lipid and nitrogen losses (g/day) for each of the control subjects and

cystic fibrosis patients. The bars represent the mean values.

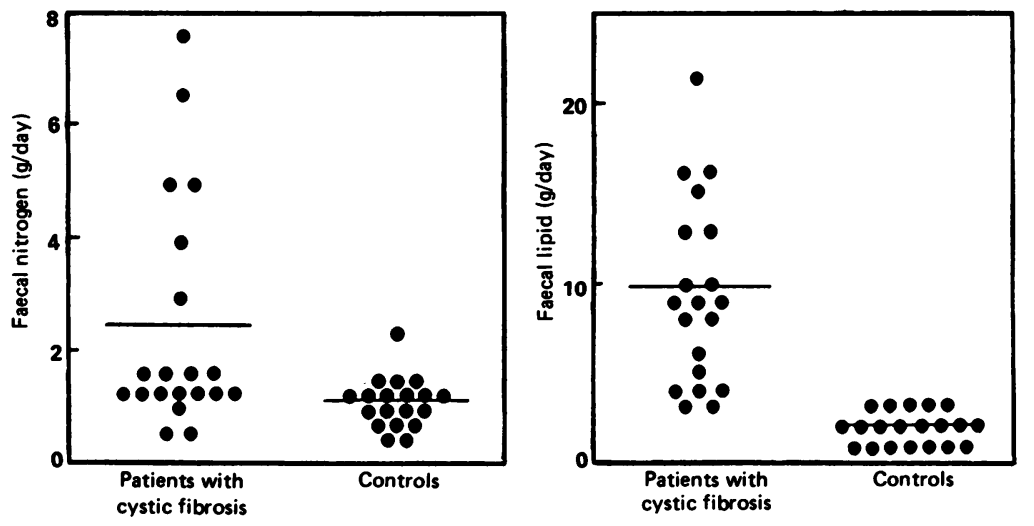




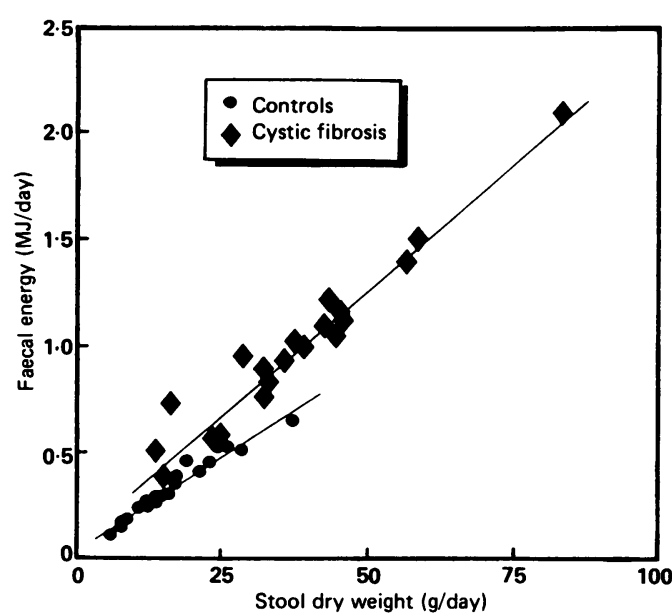

Figure 4 Stool energy losses versus stool dry weights for the control subjects and the cystic fibrosis patients.

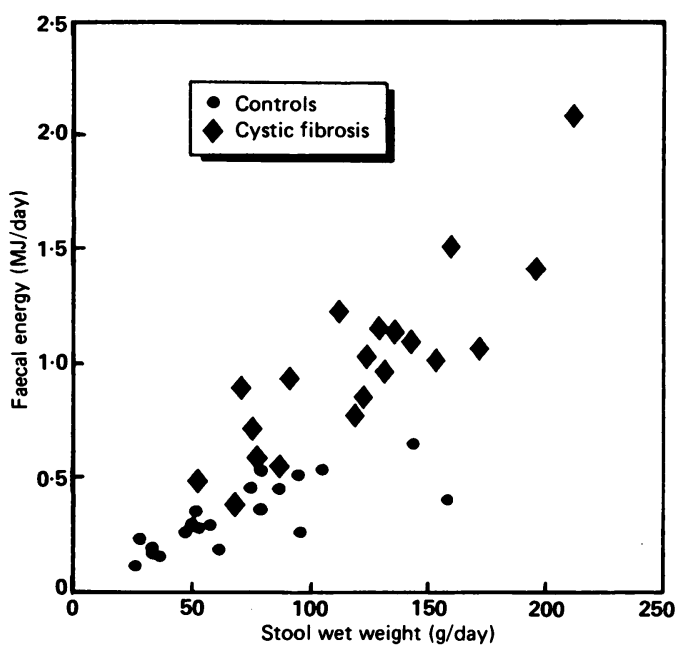

Figure 5 Stool energy losses versus stool wet weights for the control subjects and the cystic fibrosis patients.

the control subjects the regression equation describing the relationship between stool energy and dry stool weight was: stool energy (kJ/ day $)=35 \cdot 5+($ stool dry weight $(\mathrm{g} /$ day $) \times 18 \cdot 0)$; the standard error of the estimate (SEE) was $30 \cdot 6$. The regression equation for the patients with cystic fibrosis was: stool energy (kJ/day) $=78.9+($ stool dry weight (g/day) $\times 24.0)$; $\mathrm{SEE}=91 \cdot 4$. Attempts to determine whether the two lines were similar or represented two independent associations using methodology described by Zerbe $e t a l^{15}$ showed that the two lines were not statistically different. As it was not possible to differentiate between the two lines, a single expression to describe the association between stool energy and stool dry weight for both subject groups was determined; stool energy $(\mathrm{kJ} /$ day $)=-55 \cdot 7+($ stool dry weight $\times$ $\left.26 \cdot 1), S E E=96 \cdot 0, R^{2}=95 \cdot 3 \%\right)$. Stool energy per gram of dry weight of stool was significantly greater in the patients with cystic fibrosis compared with the control subjects $(27 \cdot 0(0.7) \mathrm{kJ} / \mathrm{g}$ compared with $20.4(0.4) \mathrm{kJ} / \mathrm{g} ; \mathrm{p}<0.0001)$.

More obvious differences between controls and cystic fibrosis patients were observed for the association between the energy and the wet weight of stool (controls: stool energy $(\mathrm{kJ} /$ day $)=123 \cdot 0+($ stool wet weight $(g /$ day $) \times 3 \cdot 1)$, SEE $=95 \cdot 3$; patients with cystic fibrosis: stool energy $(\mathrm{kJ} /$ day $)=68.9+$ (stool wet weight $(\mathrm{g} /$ day) $\times 7 \cdot 6), S E E=202 \cdot 0)$. Stool energy per gram of wet weight of stool was significantly greater in the patients with cystic fibrosis compared with control subjects $(8.0(0.4) \mathrm{kJ} / \mathrm{g}$ compared with $5.5(0.3) \mathrm{kJ} / \mathrm{g} ; \mathrm{p}<0.0001)$.

In healthy controls, stool bacterial mass averaged (SEM) $4.3(0.4) \mathrm{g} /$ day and was equivalent to $27 \%$ of the stool dry weight (range $17-42 \%$ ). In patients with cystic fibrosis, stool bacterial mass was three times greater $(13.4(2 \cdot 1 \mathrm{~g} / \mathrm{d}$; $\mathrm{p}<0.001$ ) and was equivalent to $35 \%$ of stool dry weight (range $19-61 \%, \mathrm{p}<0.01$ ). Determination of the energy content of the pooled stool bacterial mass samples showed an energy content per gram of bacterial mass of $20.4 \mathrm{~kJ} / \mathrm{g}$ and $23.8 \mathrm{~kJ} / \mathrm{g}$ for the pooled sample from healthy controls and patients with cystic fibrosis respectively. Using these values, the energy content of the bacteria in each of the subjects was estimated. Despite the difference in the energy content of the stool between the controls subjects and cystic fibrosis patients, a similar percentage of the energy in the stool could be attributed to the bacteria $(27 \cdot 0(1 \cdot 6) \%$ and $30.8(2 \cdot 8) \%$; this was not significant).

\section{Discussion}

The aim of the present study was to determine the magnitude and origins of stool energy losses in healthy children and in patients with cystic fibrosis on their habitual pancreatic enzyme replacement treatment. To our knowledge, this represents the first description of stool energy losses in these subject groups. In healthy children even though the energy within the stool may be relatively small, these modest losses of energy may be important as imbalances in the availability of energy from the diet may be sufficient to limit growth. In diseased states such as cystic fibrosis, where there is the maldigestion and malabsorption of lipid, protein, and carbohydrate, stool energy losses may be substantially increased. Thus if these losses in stool energy are left uncorrected, it is even more likely that growth may be restricted.

Three major components are believed to contribute to the energy within the stool: maldigested and malabsorbed dietary residue, endogenous secretions and cellular debris (for example, intestinal glycoproteins and mucopolysaccharides), and the colonic bacterial microflora. ${ }^{16}{ }^{17}$ While the relative proportions of each component may vary with dietary intake, the extent of maldigestion and malabsorption and mucus production in the gut, the relative contribution made by each of these components to stool energy losses in health and disease remains unclear. Similarly, the relative proportions of energy within the stool derived from lipid, protein, and carbohydrate have not been previously described.

Much of our understanding of stool energy, lipid, and protein losses of healthy children aged 5-16 years is derived from studies in adult subjects where stool energy was normally less than $1.0 \mathrm{MJ} /$ day and equivalent to $5 \%$ of gross 
energy intake. 'In the present study, stool energy losses in a group of normal healthy children averaged $337 \mathrm{~kJ} /$ day or $3.5 \%$ of gross energy intake. The amount of lipid and nitrogen within the stool in normal adults has been previously reported to be less than 5 g/day and $2 \mathrm{~g} /$ day respectively in normal adults. ${ }^{18}$ In the present study, stool lipid and nitrogen in this group of normal healthy children were on average $2 \cdot 2$ g/day and $1 \cdot 1 \mathrm{~g} /$ day respectively. These results for stool lipid and nitrogen are comparable with those reported in children by other workers. ${ }^{19}$

In contrast to the results for the normal healthy children, it would appear that in the group of cystic fibrosis patients on pancreatic enzyme replacement therapy who believed themselves to be comparatively asymptomatic, stool energy losses were substantially greater and were equivalent to between $5-20 \%$ of the gross energy intake. This would suggest that raised stool energy losses may continue to contribute in part towards an energy deficit that may be sufficient to limit growth or cause weight loss. We have not attempted to measure the efficacy of pancreatic enzyme replacement, although it is clear that in some of the patients with cystic fibrosis the replacement regimen may be inappropriately regulated by self titration in the absence of gastrointestinal symptoms. As the heterogeneity of the physical abnormalities of cystic fibrosis may be related to a specific gene mutation, it has been suggested that the differences in resting energy expenditure in cystic fibrosis may be associated with a defective gene product (A O'Rawe et al, personal communication). In the light of this observation, it is interesting to speculate whether the genetic nature of the disease may account for the increased stool energy losses within the present group of cystic fibrosis patients.

Although previous studies examining the efficacy of enzyme replacement have noted that stool lipid losses in patients with cystic fibrosis were approximately $12-15$ g/day ${ }^{4}$ with nitrogen losses of approximately 1-2 g/day when using pancreatic enzyme replacement therapy, ${ }^{20}$ no information on stool energy losses is available. As the stool lipid and nitogren losses in patients with cystic fibrosis in the present study were comparable with these values from earlier investigations, this would suggest that the pancreatic enzyme replacement therapy in the cystic fibrosis patients in this study reduced stool lipid and nitrogen to similar degrees. Despite this reduction in stool lipid and nitrogen, from the present study it appears that stool energy could still be equivalent to up to $20 \%$ of the gross energy intake in cystic fibrosis patients on their habitual enzyme replacement treatment.

It is also apparent that stool energy losses can not be simply related to lipid losses. While it is commonly assumed that a reduction in stool lipid losses would also represent a small amount of energy lost within the stool, it appears that cystic fibrosis patients with small lipid losses within the stool may have high stool energy losses. This would also imply that a reconsideration of the present approach to address the efficacy of pancreatic enzyme replacement by the measurement of steatorrhoea may be required.
We would suggest that the routine measurement of stool energy may provide a more accurate assessment of the extent to which pancreatic enzyme replacement treatment normalises stool energy loss in patients with cystic fibrosis. However, the facilities required to measure directly the stool energy are not generally available in clinical practice. As the regression line expressing the association between the energy present within the stool and the wet weight of the stool passes close to the origin, the use of a simple ratio based upon the slope of the line would be justified. From the data available in this study, it would appear that $8 \mathrm{~kJ}$ of energy is present in each gram of wet stool. With this information, an estimate of the extent of energy losses in the stool may be derived by simply determining the weight of stool passed each day. In the present group of cystic fibrosis patients, the $95 \%$ confidence intervals for the error in predicting stool energy loss from the wet weight of stool using a value of $8 \mathrm{~kJ} / \mathrm{g} \mathrm{stool}$ wet weight ranged from -12 to $6 \%$ (mean $-3 \%)$. This method may provide a more reliable means of evaluating the extent of maldigestion and malabsorption in patients with cystic fibrosis than the measurement of stool lipid. The apparent constancy of the energy per dry weight of stool in both the control subjects and the cystic fibrosis patients would imply that the composition of stool must remain relatively similar between individuals despite a wide range in stool energy losses.

It is generally assumed that all of the energy within the stool may be attributable to maldigested and malabsorbed dietary residue in cystic fibrosis. Stool energy losses could also be derived from endogenous secretions and cellular debris as well as the colonic bacterial microflora. Although it has not been possible to quantify directly mucus and epithelial cells losses within the faeces, this component may form a substantial part of stool energy losses in cystic fibrosis where mucus production is raised. It is comparatively simple to measure the amount of bacteria within human faeces using a novel technique described by Stephen and Cummings. ${ }^{14}$ In the present study this method has enabled the measurement of the relative contribution made by the bacteria to total energy lost within the stool in both normal healthy children and cystic fibrosis patients. To our knowledge there have been no other studies which have demonstrated the contribution made by the bacteria to the total energy lost within the stool.

Stephen and Cummings have shown that the bacterial microflora represented $55 \%$ of the total dry weight of stool in healthy male adults. ${ }^{14}$ In the present study, approximately $27 \%$ and $34 \%$ of the stool dry weight were attributable to the bacterial microflora in both the normal healthy children and patients with cystic fibrosis respectively. It appears that the bacterial microflora could account for approximately $30 \%$ of total stool energy losses in both the cystic fibrosis patients and normal healthy children. This study represents the first description of the proportion of energy within the stool which can be attributed to the bacteria. It is clear that a major component of the stool is not simply mal- 
digested and malabsorbed dietary residue but that part of the energy within the stool may be attributable to the bacterial microflora.

In conclusion, the present study has measured stool energy losses in a group of normal healthy children and patients with cystic fibrosis. The raised stool energy losses in the patients with cystic fibrosis with their habitual enzyme replacement therapy may contribute towards an energy deficit that may be sufficient to limit growth. We have shown that stool energy may be predicted from the wet or dry weight of stool. Estimating the energy within the stool each day from stool wet weight may provide a simple way of evaluating the extent to which maldigestion and malabsorption in patients with cystic fibrosis may be improved by pancreatic enzyme replacement therapy. It should not be assumed that all of this stool energy in the control subjects and cystic fibrosis patients is simply maldigested and malabsorbed dietary residue. It appears that the bacterial microflora may be a major component of the energy within the stool in both healthy children and patients with cystic fibrosis.

We thank the children and parents whose cooperation was essential for the completion of this study and for the assistance from Dr CJ Rolles, Dr S Peters, Dr V Bunker, and Mrs JE Ellis. JLM is a recipient of an MRC studentship and $S A B$ is funded by the Cystic Fibrosis Research Trust. The support of Duphar Laboratories is gratefully acknowledged.

1 Southgate DAT, Durnin JVGA. Calorie conversion factors. An experimental reassessment of the factors used in the calculation of the energy value of human diets. $\mathrm{Br} \mathcal{J} \mathrm{Nutr}$ 1970;24:517-35.

2 Di Sant'Agnese PA, Hubbard VS. The pancreas in cystic fibrosis. In: Taussig LM, ed. Cystic fibrosis. New York: Thieme-Stratton, 1984:230-95.

3 Mischler EH, Parrell S, Farrell PM, Odell GB. Comparison of effectiveness of pancreatic enzyme preparations in cystic fibrosis. Am $\mathcal{F}$ Dis Child 1982;136:1060-3.

4 Stead RJ, Skypala I, Hodson MF, Batten JC. Enteric coated microspheres of pancreatin in the treatment of cystic fibrosis: comparison with a standard enteric coated preparation. Thorax 1987;42:533-7.

5 Marr JW. Individual dietary surveys: purposes of methods. World Rev Nutr Diet 1971;13:105-64.

6 Paul AA, Southgate DAT. McCance and Widdowson's the composition of foods. London: HMSO, 1978.

7 Tan SP, Wenlock RW, Buss DH. Second supplement to McCance and Widdowson's the composition of foods: immigrant foods. London: HMSO, 1985.

8 Holland B, Unwin ID, Buss DH. Third supplement to McCance and Widdowson's the composition of foods: cereals and cereal products. Nottingham: The Royal Society of and cereal products. Nottingham: The Royal Society of Food, 1988 .

9 Holland B, Unwin ID, Buss DH. Fourth supplement to McCance and Widdowson's the composition of foods: milk products and eggs. Cambridge: The Royal Society of Chemistry and The Ministry of Agriculture, Fisheries and Food, 1989.

10 Merrill AL, Watt BK. Energy value of foods . . . basis and derivation. United States Department of Agriculture handbook. 1955:74.

11 Gompertz SM, Sammons HG. The origin of faecal lipids. The composition of faecal fats. Clin Chim Acta 1963;8:591-603.

12 Van de Kamer JH, ten Bokkel Huinink H, Weyers HA. Rapid method for determination of fat in feces. 7 Biol Chem 1949;177:347-55.

13 Miller PS, Payne PR. A ballistic bomb calorimeter. Brf Nutr 1959;13:501-8.

14 Stephen AM, Cummings JH. The microbial contribution to human faecal mass. F Med Microbiol 1980;13:45-56.

15 Zerbe GO, Archer PG, Banchero N, Lechner AJ. On comparing regression lines with unequal slopes. Am $\mathcal{f}$ Physiol paring regression lin.

16 Lusk G. The elements of the science of nutrition. Philadelphia: Saunders, 1906 (4th ed 1928). London: Academic Press, 1976:46-60. (Reprint.)

17 Cummings JH. Constipation, dietary fibre and the control of large bowel function. Postgrad Med F 1984;60:811-9.

18 Wollaeger EE, Comfort MW, Osterberg AE. Total solids, fat and nitrogen in the feces III. A study of normal persons. Gastroenterology 1947;9:272-83.

19 Schmerling DH, Forrer JCW, Prader A. Fecal fat and nitrogen in healthy children and in children with malabsorption or maldigestion. Pediatrics 1970;46:690-5.

20 Beverley DW, Kelleher J, MacDonald A, Littlewood JM, Robinson T, Walters MP. Comparison of four pancreatic extracts in cystic fibrosis. Arch Dis Child 1987;62:564-8.

Pylorus 6 balloonists 0

If balloon dilatation can be used so successfully in the heart and blood vessels and, indeed, in almost any tubular structure, why not in pyloric stenosis? Canadian workers recently tried it and unfortunately scored six own goals.

A report from Halifax, Nova Scotia (Hayashi et al, Fournal of Pediatric Surgery 1990;25:1119-21) describes the use of a balloon in six babies with hypertrophic pyloric stenosis. In order to check on the safety of the procedure it was done with direct observation of the pylorus at operation. After a right upper quadrant transverse incision the balloon was passed through the mouth and its position in the pylorus was checked by the surgeon before it was inflated.

A perforation of the mucosa occurred in one baby and in the others the split in the pyloric muscle was deemed inadequate. In three there was no split, in two it was partial, and the only child with a complete split was the one with the perforation.

Hard luck! It looks as if Ramstedt's operation will have to stay, at least for the time being. 